Showa Univ. J. Med. Sci. 6(1), 105 109, June 1994

\title{
Original
}

\section{The Interscalar Septum of the Human Cochlea}

\author{
Atomu Suzuki and Yasuya Nomura
}

\begin{abstract}
The interscalar septum (ISS) between the apical and middle turns was dissected out of the human cochlea and morphologically studied. The ISS is a thin bony plate, in which run the radiating arterioles to the middle turn and the venules from the lower spiral ligament of the apical turn. The radiating arterioles are located within the fairly large perivascular space. Melanocytes with irregular or granular shapes adhere to the wall of the radiating arterioles. These cells also adhere to the wall of the perivascular space without touching the arteriole wall. Strands, which are possibly remnants of atrophied blood vessels are observed within the space. An avascular channel is also observed. Indian ink injected into the modiolus reaches the upper spiral ligament via the perivascular space of the radiating arteriole. It is possible that cerebrospinal fluid reaches the cochlear lateral wall through the same space to participate in formation of the perilymph.
\end{abstract}

Key words: interscalar septum, perivascular space, perilymph, cerebrospinal fluid, melanocyte

\section{Introduction}

The interscalar septum (ISS) separates the cochlear turns and contains blood vessels to and from the lateral wall of the cochlea. There are many spaces found in cross-sections of the human ISS. This paper reports a study of the anatomy of the ISS, particularly with regard to the cerebrospinal fluid pathway to the membranous cochlea in humans.

\section{Materials and Methods}

Twenty human temporal bones of different ages were used in this series of studies. Of these temporal bones, 15 were fixed in $10 \%$ formalin, decalcified using $5 \%$ trichloroacetic acid solution, washed in tap water, neutralized in 5\% sodium sulfate solution and washed again in tap water. The ISS between the apical and middle turns was dissected out from the decalcified temporal bone under a surgical microscope. ISS specimens were observed as surface preparations with or without staining after dehydration and mounting in Malinol. Staining solutions were hematoxylin and eosin (HE), Azan, Trichrome and van Gieson's. Two fresh temporal bones were used for electron microscopic study of the pigmentary cells. Formalin-fixed ISS specimens were post-fixed in $1 \% \mathrm{OsO}_{4}$ solution. Sections for transmission electron microscopy were double-stained with uranyl acetate and lead citrate and examined under a Hitachi-H800 electron microscope (Hitachi: Tokyo, Japan).

One milliliter of Indian ink was injected through the fundus of the internal auditory meatus into the modiolus of five fresh temporal bones to verify connection of the bony channels

Department of Otolaryngology, Showa University School of Medicine, 1-5-8 Hatanodai, Shinagawa-ku, Tokyo 142, Japan. 
within the ISS to the spiral ligament. The temporal bones were either dissected out for surface preparation or sectioned for HE staining after decalcification and paraffin embedding.

\section{Results}

The ISS between the apical and middle turns is less than $100 \mu \mathrm{m}$ thick, and can be observed in transparent specimens. The pigmentary cells are abundant near the modiolus in unstained specimens. Parallel linings of the cells, radiating laterally from the modiolus, indicate courses of the bony channels and radiating arterioles to the cochlear lateral wall (Fig. 1).

Spaces within the ISS are mainly perivascular channels, in which run the radiating arterioles. The channels are distributed radially from the modiolus (Fig. 2). The diameters of the channels are 50-75 $\mu \mathrm{m}$, whereas those of the arterioles are 9-16 $\mu \mathrm{m}$ (Fig. 3). Measurements were performed on surface preparations. Strands, possibly remnants of blood
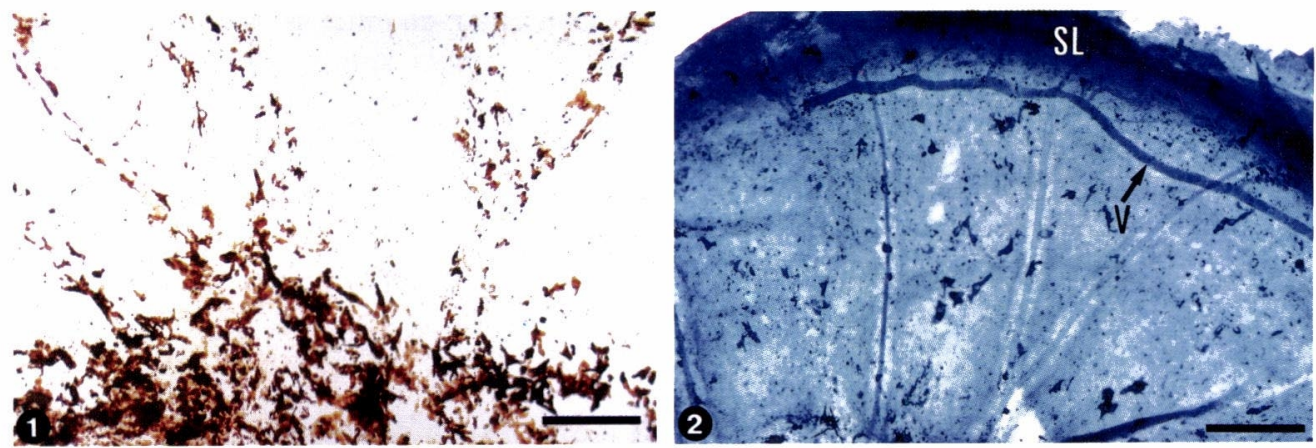

Fig. 1. Pigmentary cells are abundant near the modiolus (lower part of photograph). Parallel arrangement of pigmentary cells indicates the presence and direction of channels. Unstained surface preparation. 50-year-old male. Scale: $200 \mu \mathrm{m}$.

Fig. 2. Radially arranged bony channels with radiating arterioles. A venule (V) runs spirally, close to the lower spiral ligament (SL). Surface preparation: Azan stain. 81-year-old female. Scale: $200 \mu \mathrm{m}$.
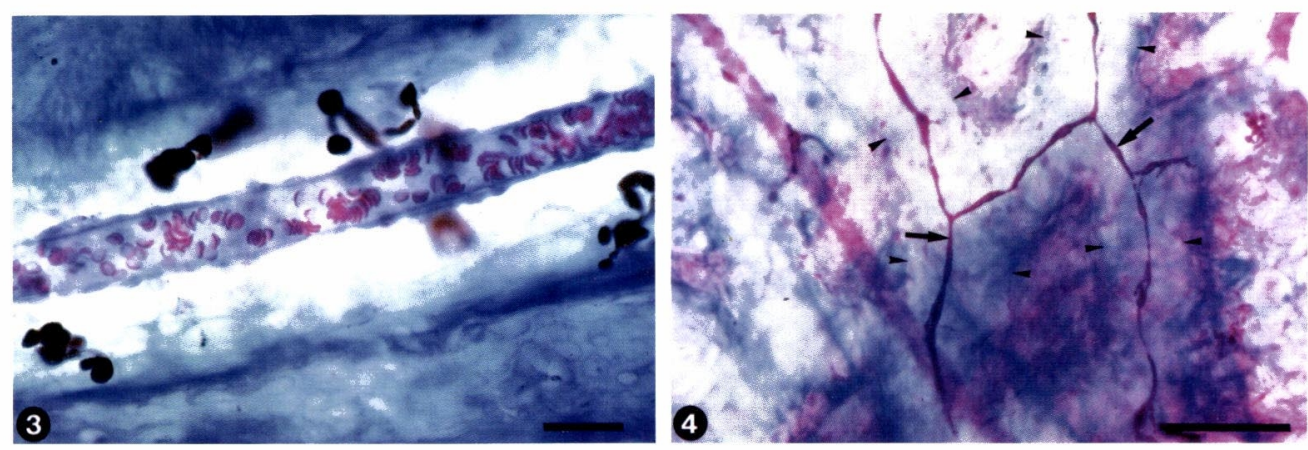

Fig. 3. Radiating arteriole running within the bony channel. Pigmentary cells are scattered in the channel. Diameter of the arteriole is $15 \mu \mathrm{m}$, and that of the channel is $55 \mu \mathrm{m}$. Surface preparation: Azan stain. 71-year-old female. Scale: $1 \mu \mathrm{m}$.

Fig. 4. Strands (large arrows) in the bony channel (small arrows). Surface preparation: Azan stain. 83-year-old female. Scale: $100 \mu \mathrm{m}$. 

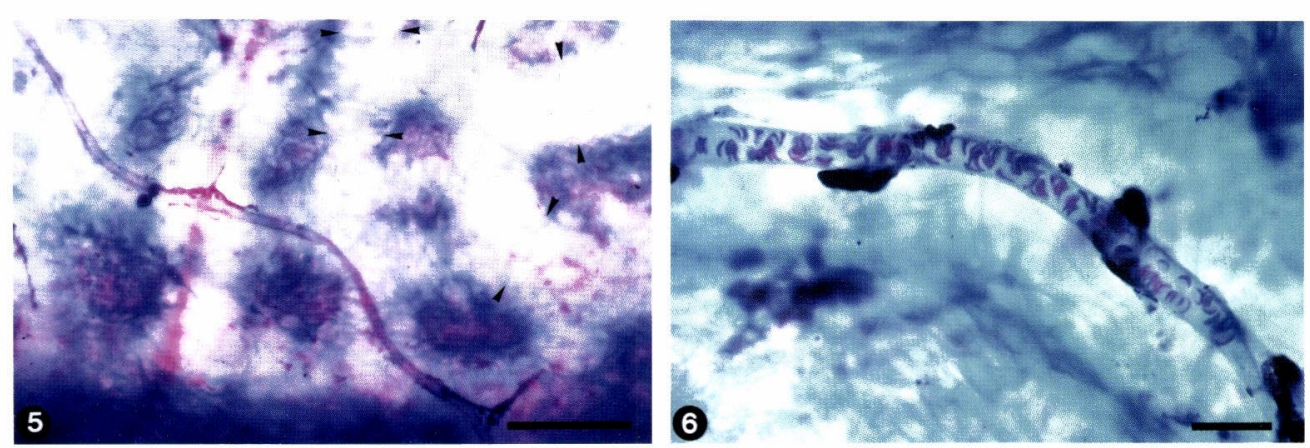

Fig. 5. Avascular channel (arrows). Surface preparation. Azan stain. 83-year-old female. Scale: $100 \mu \mathrm{m}$.

Fig. 6. Pigmentary cells adhering to the radiating arteriole. Surface preparation. Azan stain. 71-year-old female. Scale: $1 \mu \mathrm{m}$.
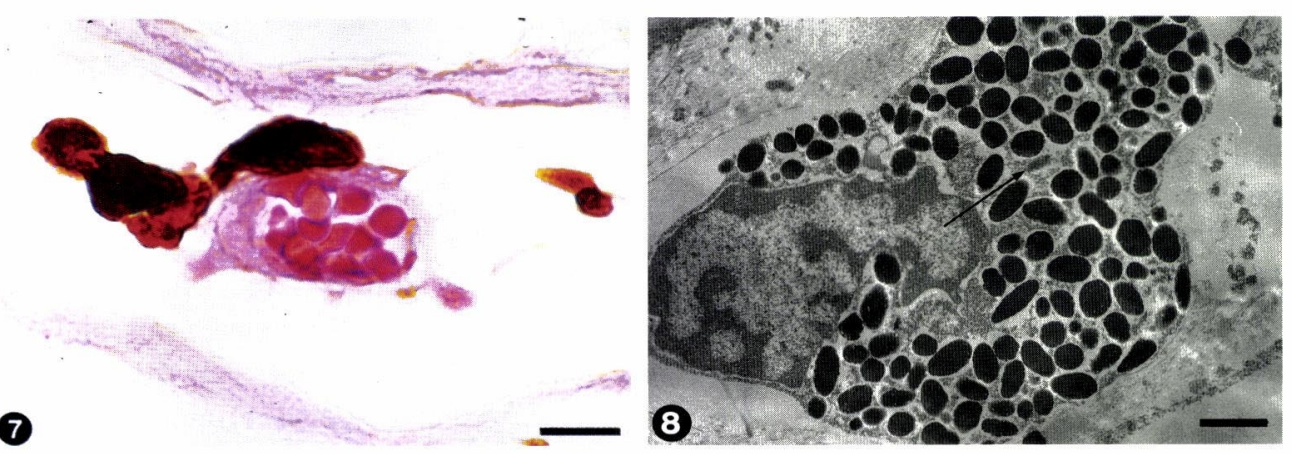

Fig. 7. Pigmentary cell on a radiating arteriole. H-E stain. 81-year-old female. Scale: $1 \mu \mathrm{m}$.

Fig. 8. Pigmentary cell containing many melanosomes and premelanosomes (arrow). 61year-old male. $\times 12,000$. Scale: $1 \mu \mathrm{m}$.
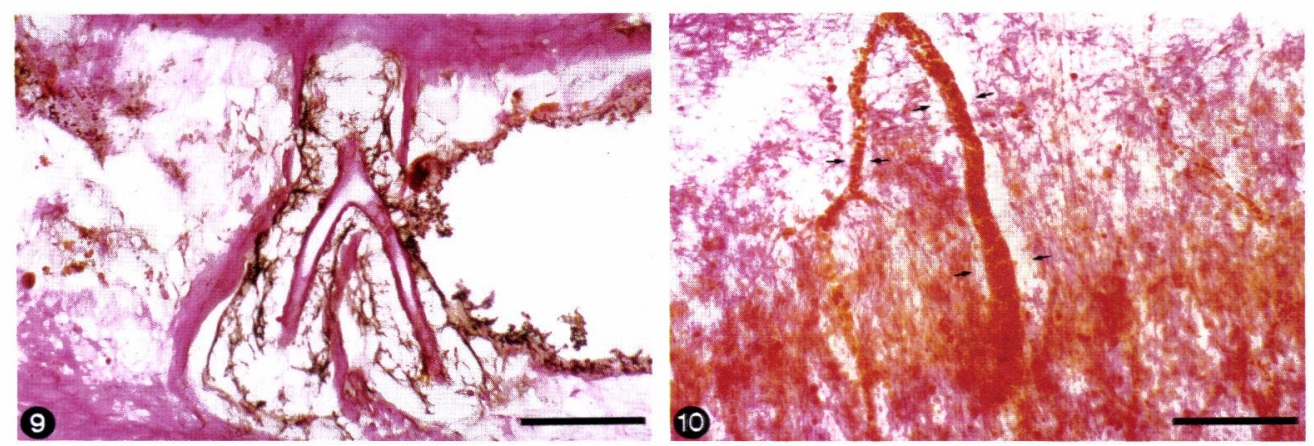

Fig. 9. Bony canal protruding into the upper spiral ligament from the interscalar septum. The inside of the bony canal is stained by Indian ink. H-E stain. 61-year-old male. Scale: $100 \mu \mathrm{m}$.

Fig. 10. Radiating arterioles in the upper spiral ligament. The perivascular space is observed (arrows). Surface preparation. van Gieson stain. 84-year-old male. Scale: $100 \mu \mathrm{m}$. 

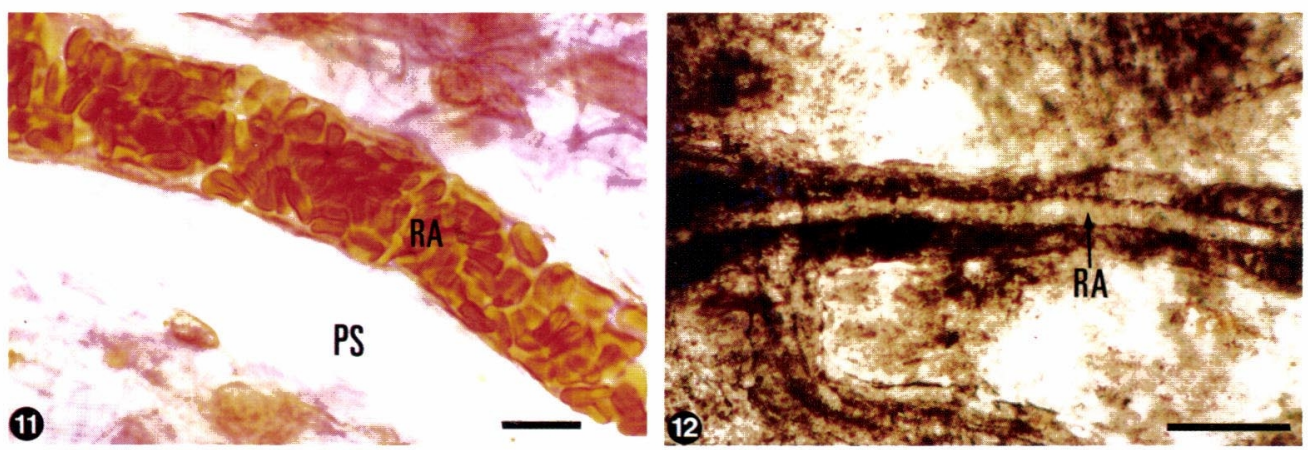

Fig. 11. Higher power view of the radiating arteriole (RA) and perivascular space (PS). Surface preparation. van Gieson stain. 84-year-old male. Scale: $1 \mu \mathrm{m}$.

Fig. 12. Indian ink in the channel around the radiating arteriole (RA). Unstained surface preparation. 68-year-old male. Scale: $100 \mu \mathrm{m}$.

vessels, were occasionally found in the channels (Fig. 4). Avascular channels were also observed (Fig. 5). The course of the venules was not radial. They ran spirally, starting from the lower spiral ligament of the apical turn. There were also perivascular spaces around the venules. The pigmentary cells were of irregular or granular shape and adhered to the arterial walls (Figs. 6, 7). However, they were also found scattered within the bony channels, not in contact with the arterioles (Fig. 3).

Electronmicroscopic observation of the pigmentary cells revealed the presence of many melanosomes and premelanosomes within the cells (Fig. 8). At the junction of the ISS and the spiral ligament, a thin bony canal was found protruding as an outlet of the channel into the spiral ligament (Fig. 9). There were distinct perivascular spaces around the radiating arterioles in the upper spiral ligament (Figs. 10, 11). This continued from the perivascular space within the ISS.

Indian ink injected into the modiolus was found in the perivascular space of the ISS and spiral ligament (Figs. 9, 12), and this was found in all temporal bones studied.

\section{Discussion}

In the second trimester of human embryogenesis, the perilymphatic spaces of the cochlear turns are separated by thick mesodermal tissue which contains relatively large blood vessels from the anlage of the modiolus. There are no spaces around the vessels. Later, this region develops into the ISS.

The arterial supply to the spiral ligament and stria vascularis is derived from the radiating arterioles running over the roof of the scala vestibuli within the ISS. The venous return from the spiral ligament and stria vascularis is via the collecting venules of the lower spiral ligament. These vessels also traverse the ISS and join the spiral modiolar vein. Therefore, the ISS is an important structure for maintenance of cochlear function. A dehiscence of the ISS, termed "scala communis"1) or "interscalar septum defect" ${ }^{2}$, has been reported in anomalous human temporal bones, most commonly in the Mondini anomaly.

The bony channels seem to develop as perivascular spaces of radiating arterioles. The channels are devoid of lining cells, and are fairly large in diameter relative to the arterioles.

The melanocytes are most numerous in the cochlea, their density being greatest on the 
bony wall of the modiolus and on the osseous spiral lamina ${ }^{3,4)}$. The role of melanocytes in the bony channel is not known. Some authors are of the opinion that the melanocytes may have a vasomotor function ${ }^{3,5)}$. They adhere to both the vessel walls and the inner walls of the channels. The arterioles seem to run in the middle of the channels, unattached to the walls. The radiating arterioles may be suspended in the bony channels partly by thin fibrous strands.

Some bony channels are avascular and these occasionally contain strands. The channels could possibly develop with blood vessels, but atrophy of the blood vessels may occur with aging, resulting in the formation of strands and later avascular channels. Johnsson describes similar findings in the human osseous spiral lamina"), and termed his finding "intervascular strands", which probably consist of remnants of vessel walls.

Indian ink injected into the modiolus passes through the perivascular space and reaches the spiral ligament. This indicates the possibility that cerebrospinal fluid can reach the spiral ligament through the channels.

There are several possibilities for the source of perilymph, (1) ultrafiltrate of blood in the inner ear, (2) a direct product of cerebrospinal fluid, and (3) arising from endolymph. The cochlear aqueduct is commonly accepted as a connection between the perilymph and cerebrospinal fluid. However, there is doubt regarding the active flow of cerebrospinal fluid into the cochlea. According to Silverstein and colleagues ${ }^{i)}$, blockage of the cochlear aqueduct did not alter concentrations of sodium, potassium, or glucose in the perilymph.

Müsebeck demonstrated the presence of acid mucopolysaccharide in "Lymphspalten" of the modiolus of the guinea pig $^{8)}$, and suggested a regulatory function of the inner ear fluid.

In conclusion, although it is not clear how cerebrospinal fluid participates in the formation of perilymph, it can reach the upper spiral ligament through the bony channel of the ISS.

\section{References}

1) Alexander G: Zur Pathologie und Pathologischen Anatomie der kongenitalen Taubheit. Arch Ohren Nasen Kehlkopfheilkd, 61: 183-219 (1904)

2) Altmann F: Malformations, anomalies and vestigial structures of the inner ear. Arch Otolaryngol, 57: 591-602 (1953)

3) Savin C: The blood vessels and pigmentary cells of the inner ear. Ann Otol Rhinol Laryngol, 74: 611-622 (1965)

4) Inoue K: A morphological and biochemical study of melanin in the inner ear. J Tokyo Women's Med College, 61: 430-438 (1991)

5) Johnsson L-G: Cochlear blood vessel pattern in the human fetus and postnatal vascular involution. Ann Otol Rhinol Laryngol, 81: 22-90 (1972)

6) La Ferriere KA, Arenberg IK, Howkins JE Jr and Johnsson LG: Melanocytes of the vestibular labyrinth and their relationship to the microvasculature. Ann Otol Rhinol Laryngol, 83: 685-694 (1974)

7) Silverstein H, Davies D and Griffin W Jr: Cochlear aqueduct obstruction change in perilymph biochemistry. Ann Otol Rhinol Laryngol, 78: 532-541 (1969)

8) Müsebeck K: Die perivasculären Lymphspalten des Innenohres. Arch Ohren Nasen Kehlkopfheilkd, 184: 560-570 (1965) 